\title{
Modeling Multiphase Effects on Pressure Oscillations in Solid Propulsion
}

\author{
M. Grossi* D. Bianchi ${ }^{\dagger}$ and B. Favini ${ }^{\ddagger}$
}

In this work multiphase effects on pressure oscillations within solid rocket motors are addressed with particular focus on thermoacoustics. A mathematical and numerical approach is described and implemented in an already existing CFD in-house code, taking care of employing the most simplified physical describing tool suite. In order to validate such methodology, numerical simulations have been performed on simplified geometries known in literature to be prone to develop pressure fluctuations due multiphase effects. The presented solutions show actually an instable behaviour, whose thermoacoustic nature is proved by the presence of its characteristics key features and by Rayleigh criterion analysis. Such result ensures the model validation, which is the preliminary and obligatory step of a work-in-progress project aimed to improve the understanding about pressure oscillations in solid rocket motors.

\section{Nomenclature}

$\begin{array}{ll}B & =\text { Spalding number } \\ C_{d} & =\text { drag coefficient } \\ D & =\text { particle diameter } \\ D_{\text {inj }} & =\text { aluminum agglomerate diameter at injection } \\ D_{\text {res }} & =\text { aluminum oxide particle diameter } \\ \vec{F}_{\text {drag }} & =\text { drag force vector } \\ L_{v} & =\text { latent heat of vaporization } \\ n_{p} & =\text { number of particles per unit volume } \\ N u & =\text { Nusselt number } \\ P r & =\text { Prandtl number } \\ Q_{A l} & =\text { aluminum heat of reaction per unit mass } \\ Q_{c o n v} & =\text { convective heat transfer } \\ R e & =\text { particles Reynolds number } \\ S h & =\text { Sherwood number } \\ T & =\text { temperature } \\ T_{s a t} & =\text { particles burning temperature } \\ \vec{u} & =\text { velocity vector } \\ \alpha_{p} & =\text { particles volume fraction } \\ k_{i n j} & =\text { particles mass loading } \\ \lambda_{g} & =\text { gas thermal conductivity } \\ \mu & =\text { dynamic viscosity } \\ \rho & =\text { density } \\ \rho_{A l} & =\text { aluminum density } \\ \dot{\omega}_{p} & =\text { mass release rate for aluminum combustion }\end{array}$

\section{Subscripts}

$g=$ gaseous phase propriety

$p=$ condensed phase propriety

\footnotetext{
*Ph.D. Student, Department of Mechanical and Aerospace Engineering, marco.grossi@uniroma1.it

$\dagger$ Ph.D. Associate Professor, Department of Mechanical and Aerospace Engineering, daniele.bianchi@uniroma1.it

$\doteqdot$ Associate Professor, Department of Mechanical and Aerospace Engineering, bernardo.favini@uniroma1.it
} 


\section{Introduction}

$\mathrm{T}^{\mathrm{N}}$ a recent survey on combustion instabilities occurring in rocket motors [1], four chief mechanisms, represented in Fig,1, may lead to flow instabilities in solid rockets: pressure and velocity coupling, hydrodynamic instabilities (i.e. vortex shedding) and distributed combustion of fuel particles.

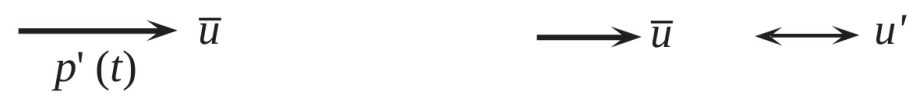

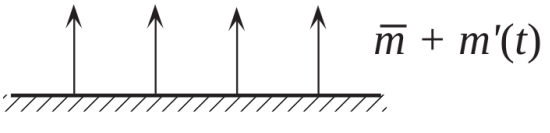

(a) Pressure coupling.

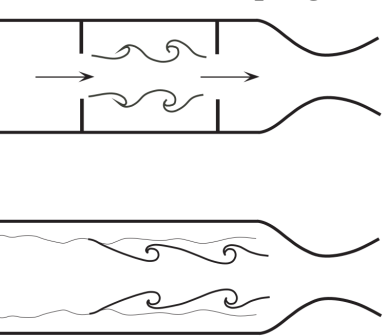

(c) Vortex shedding.

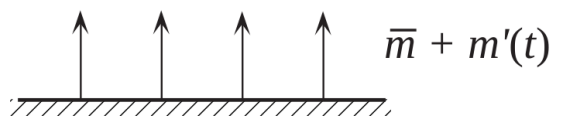

(b) Velocity coupling.

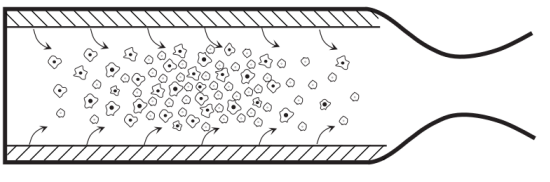

(d) Distributed combustion.

\section{Fig. 1 The four possible mechanisms for combustion instabilities in solid propellant rockets [1].}

The first two phenomena are usually present in small motors, as those used in tactical propulsion, where instabilities are usually due to grain propellant combustion response [2]. On the contrary, for large motors, PO signatures encourage to rely mainly on hydrodynamic instabilities, as in Ariane 5 P230 [3, 4], US Space Shuttle boosters [5] and the Titan family rockets [6, 7]. In such cases, large scale vortical structures are generated by the interaction of the flow with obstacles (Obstacle Vortex Shedding) or a sharp change of the propellant geometry (Angular Vortex Shedding) or by propellant gas injection (Parietal Vortex Shedding) and pressure oscillations production is the result of vortexes deformation and destruction while they are carried towards the nozzle motor exit. Alongside with these well known instabilities sources, the dynamics of residual combustion relatively far from the burning surface, most likely associated with aluminum or other metal fuel additives not completely burned at the surface, has recently been considered as an additional, but less studied, source of pressure oscillations. Indeed a coupling between acoustics and the combustion of aluminum has early been suspected to explain certain SRM unsteady behavior, as the one observed in U.S. Army missile Sentry [8]. In Europe the development of aft-finocyl SRMs exploited in the VEGA launchers family and Ariane 6 has brought renewed interest in pressure oscillations issue, due to experimental data concerning the VEGA first stage P80 which show sustained low level pressure fluctuations during the first half of the operative time [9]. Even though the strongly reduced PO levels, the same behaviour has been spotted in the French demonstrating motor POD-Y [10, 11], built and fired during the ongoing research on Europe next generation motor P120C. Notwithstanding the conclusions provided by the lone work [12] on multi-phase reagent flow simulation on aft-finocyl SRM do not address the presence of any thermoacoustic feedback loop as a PO source, in the current state-of-the art [13], this last is thought to have an important role in PO generation and characterization along with the well known hydrodynamic instabilities.

Aluminum particles are added to solid composite propellants with the aim to increase performances and reduce high frequencies instabilities. The proportion of aluminum can reach the value of $20 \%$ of the overall propellant mass in typical Al/AP/HTPB mixture. Such a configuration may yield to the formation of large aluminum globules resulting from the agglomeration of the initial population [14, 15]. Since the diffusive nature of aluminum combustion, this last is strongly influenced by the environment flow condition in which the reaction is taking place, especially regarding convection conditions: the effect of a relative velocity between the droplet and the conveying gas is to increase the evaporation or combustion rate. From such interaction arises the possibility of a pure thermoacoustic feedback loop: an acoustic disturbance triggers a combustion rate increasing which, in turn, enhances flow instability. Anyway, despite convection effects are known since first works on aluminum combustion [16, 17], the onset of a thermoacoustic coupling 
involving an oscillatory gaseous field and particles combustion has been firstly addressed by Raun and Beckstead in 1993 [18] and then very recently studied and analyzed in French literature [19-23]. Nevertheless the way aluminum droplet combustion couples with the acoustic field remains not fully understood and the research about this topic represents an ongoing challenge especially when other kinds of instability sources can be meanwhile present.

At the Department of Aerospace and Mechanical Engineering (DIMA) in Sapienza University of Rome, the authors have been working on pressure oscillations in SRMs characterized by aft-finocyl geometries, employing a Q1D, mono-phase formulation named AGAR [24, 25] in which instability source is strictly related to vortex-shedding phenomena. Thermoacoustics is not directly evaluated, but roughly taken into account thanks to proper calibration parameters which have allowed to provide a reconstruction of VEGA P80 and POD-Y SRMs PO envelopes [26]. Anyway experimental data and new insights in the state-of-the-art, have prompted the authors to look for an enriched physical model to deal with SRMs instability. Such necessity leads to find a model able, on one hand, to cope with propellant combustion and multiphase effects in a more direct way and, to the other one, to be embedded with the Q1D formulation of AGAR. Nevertheless the development of such reduced model when dealing with this physical phenomenology is not straightforward, hence a preliminary multidimensional analysis aimed to the understanding of the key points of thermoacoustic instabilities is required. In DIMA framework, multidimensional simulations in aft-finocyl SRMs have already been performed with several approaches and methodologies, without, anyway, never addressing theromoacoustics modeling. Laureti et al. [27] used a single-phase ILES approach to perform analysis on hydrodyanimc instabilities. Similar methods were employed in the work of Di Mascio et al. [28] in VEGA Zefiro 23 flowfield reconstruction. Only the work of Attili et al. [29] applied a multiphase reagent approach to SRMs geometries, nevertheless the aluminum combustion model did not account for convective effects on particles chemical reaction, nullifying any possibility to actually catch pressure instabilities driven by condensed phase distributed combustion.

This work has the task to present and validate a mathematical and numerical code able to cope with multiphase flow in SRMs with particular focus on thermoacoustics phenomena, employing the most simplified physical describing tool suite. Moreover it represents, in the DIMA framework, the first and preliminary step in a more challenging work-in-progress project aimed to a twofold task: performing multiphase simulation on 3D complex geometries of in-operation SRMs accounting for the two main PO sources and provide the physical basis and understanding to build up a reduced model focused on thermoacoustics to be embedded in the already existing Q1D model for PO analysis.

\section{Multiphase Modeling}

The model described hereafter follows the one presented by Marble [30], which is based on a multidimensional formulation applied to a set of equations able to solve gaseous and condensed phases behaviour within motor chambers. Since one of the aim of this work is to reduce the number of elements necessary to investigate thermoacoustics phenomena, an inviscid Euler set of equations is solved instead of the more complex and burdensome Navier-Stokes one to provide the numerical solution of the gaseous phase. As for the particles one, a pressure-less fully Eulerian model is exploited, with condensed phase supposed dilute (volume fraction $\alpha_{p}=\frac{\rho_{p}}{\rho_{A l}} \ll 1$ ), monodisperse (i.e., a single class of injected particles) and assumed to be spherical. The Eulerian approach has been preferred to the Lagrangian one due to its lower computational cost [19, 31].

The aforementioned multiphase model has been introduced in an in-house single-phase code employed at DIMA in several contexts as liquid rocket engines, hybrid rocket engines, hypersonics and nozzle ablation computations.

The governing equations read:

$$
\frac{\partial}{\partial t}\left(\begin{array}{l}
W_{g} \\
W_{p}
\end{array}\right)+\nabla\left(\begin{array}{l}
F_{g} \\
F_{p}
\end{array}\right)=\left(\begin{array}{l}
S_{g} \\
S_{p}
\end{array}\right)
$$

where $W$ denotes the conservative variable vector, $F$ is the flux vector, and $S$ is the two-way source term vector. Subscripts $g$ and $p$ stand for the gas phase and particle phase, respectively. Pressure-less dynamics equations for the particle phase state:

$$
W_{p}=\left(\begin{array}{c}
\rho_{p} \\
\rho_{p} \vec{u}_{p} \\
\rho_{p} e_{p} \\
n_{p}
\end{array}\right) \quad, \quad F_{p}=\left(\begin{array}{c}
\rho_{p} \vec{u}_{p} \\
\rho_{p} \vec{u}_{p} \otimes \vec{u}_{p} \\
\rho_{p} \vec{u}_{p} e_{p} \\
n_{p} \vec{u}_{p}
\end{array}\right) \quad, \quad S_{p}=-\left(\begin{array}{c}
\dot{\omega}_{p} \\
\dot{\omega}_{p} \vec{u}_{p}-\vec{F}_{\text {drag }} \\
\dot{\omega}_{p} e_{p}-\vec{F}_{\text {drag }} \cdot \vec{u}_{p}+\dot{\omega}_{p} L_{v}-Q_{\text {conv }} \\
0
\end{array}\right)
$$


where $\rho_{p}$ is the particle density (varying due to volume fraction modification), $\vec{u}_{p}$ is the particle velocity vector, $e_{p}$ is the particle total energy, and $n_{p}$ is the number of particles per unit volume. Note that the equation on $n_{p}$ is a simple transport equation because neither coalescence nor break-up is currently considered. The main advantage of using four equations, instead of three valid for mass, momentum and energy balance law, relies on the capability of representing the condensed phase dimension evolution due to burning reactions. Solving in time the density and number of particles equations allow to directly compute the particle diameter $D$, thus not explicitly tracked, as:

$$
D=\left(\frac{6 \rho_{p}}{\pi \rho_{A l} n_{p}}\right)^{\frac{1}{3}}
$$

with $\rho_{A l}$ the material aluminum density assumed constant.

\section{A. Aluminum Combustion Modeling}

Several models may be applied to evaluate the aluminum combustion. Besides the simple $D^{2}$ law early proposed by Glassman [32, 33] for metal combustion, in literature two main approaches, actually more precise and carefully designed for aluminum combustion, are adopted (with their several derivations): Law's [34] and Hermsen's [35]. Nevertheless, despite of the basic model employed, in order to provide a coupling between acoustic velocity field and combustion heat release, it is of paramount importance the introduction of elements able to address convective effects.

In order to take the model as simple as possible, pursuing the aim to find the minimum requirements to address thermoacoustics, in this work mass release due to aluminum combustion follows a classical $D^{2}$ law and the reaction products are supposed to be chemically equal to the environment. Mass rate is thus computed, with unity Lewis number assumption, as:

$$
\dot{\omega}_{p}=\pi D n_{p} \log (1+B) S h
$$

with $B$ representing the Spalding number[36] whereas $S h$ is the Sherwood number. The modeling of this last is fundamental since it involves flow convection effects on combustion mass release thanks to several relationships. One of the most employed [19, 37, 38] is the one proposed firstly by Frössling but usually attributed to Ranz-Marshall which states an empirical law for the Sherwood number [39]:

$$
S h=2\left(1+0.33 \operatorname{Re}^{0.5} \operatorname{Pr}^{0.33}\right)
$$

in which appears the Reynolds number built on the particle diameter:

$$
R e=\frac{\rho_{g}\left|\vec{u}_{g}-\vec{u}_{p}\right| D}{\mu}
$$

Particles combustion is defined as a fully isothermal process, therefore the convective heat streaming from gas to particles is wholly employed to allow particle vaporization $\left(Q_{c o n v}=\dot{\omega}_{p} L_{v}\right)$. Once a certain threshold diameter $D_{r e s}$ is reached, burning is inhibited for addressing oxide cap development and pure heat exchange can actually occur. Note that all combustion heat coming out from the aluminum chemical reaction is only addressed to the gas phase, therefore in the current model gas and condensed phases source terms do not respect conservation principles until particles burn-out. For sake of clarity, source terms are explicitly written for both phases and combustion occurring:

- Burning Particles

$$
S_{p}=-\left(\begin{array}{c}
\dot{\omega}_{p} \\
\dot{\omega}_{p} \vec{u}_{p}-\vec{F}_{d r a g} \\
\dot{\omega}_{p} e_{p}-\vec{F}_{d r a g} \cdot \vec{u}_{p} \\
0
\end{array}\right) \quad, \quad S_{g}=\left(\begin{array}{c}
\dot{\omega}_{p} \\
\dot{\omega}_{p} \vec{u}_{p}-\vec{F}_{d r a g} \\
\dot{\omega}_{p} e_{p}-\vec{F}_{d r a g} \cdot \vec{u}_{p}+\dot{\omega}_{p} Q_{A l}
\end{array}\right)
$$

\section{- Inert Particles}

$$
S_{p}=-\left(\begin{array}{c}
0 \\
-\vec{F}_{d r a g} \\
-\vec{F}_{d r a g} \cdot \vec{u}_{p}-Q_{\text {conv }} \\
0
\end{array}\right) \quad, \quad S_{g}=-S_{p}
$$


Finally drag force and heat exchange formulations are reported. The drag force vector $F_{d r a g}$, with the Clift correlation [40] for the drag coefficient $C_{d}$, reads:

$$
\begin{aligned}
\vec{F}_{\text {drag }} & =\frac{1}{8} \pi D^{2} n_{p} \frac{C_{d}}{1+B} \rho_{g}\left|\vec{u}_{g}-\vec{u}_{p}\right|\left(\vec{u}_{g}-\vec{u}_{p}\right) \\
C_{d} & =\max \left(0.44, \frac{24}{R e}\left(1+0.15 \operatorname{Re}^{0.687}\right)\right)
\end{aligned}
$$

As for heat transfer, only convection is accounted for (no radiative transfer):

$$
\begin{gathered}
Q_{\text {conv }}=\pi \lambda_{g} D n_{p} N u\left(T_{g}-T_{p}\right) \\
N u=2+0.6 \operatorname{Re}^{0.5} \operatorname{Pr}^{0.333}
\end{gathered}
$$

\section{B. SRM Propellant Combustion}

Since the application of such modeling is mainly oriented toward SRM numerical simulation, particular focus is required when dealing with the in-flow boundary condition applied to the grain burning surface. Propellant combustion is modeled by an injection boundary condition with an imposed propellant mass rate $\dot{m}$ normal to surface and a stagnation temperature, for gas and particles. Let $k_{i n j}$ be the particle (i.e., aluminum agglomerate) mass fraction at the propellant surface; the particle mass rate is evaluated as $k_{i n j} \dot{m}$, and the gas mass rate is consequently $\left(1-k_{i n j}\right) \dot{m}$. The remaining part of aluminum mass loading which does not provide agglomerates is supposed to burn very closely to the surface and considered in the gaseous phase.

The propellant mass rate is considered as pressure-dependent and given by a De Saint-Robert law $\left(\dot{m} \propto a p_{c}^{n}\right)$. Particles are assumed to be injected at burning conditions $\left(T_{p}=T_{s a t}\right)$ and in kinetic equilibrium with the gas flow, whereas injection gas temperature is changed depending on the exact value of $k_{i n j}$ in a way that the temperature reached after finalisation of aluminum combustion is equal to adiabatic flame temperature for a classical aluminized propellant. Particles initial diameter requirement provides the $n_{p}$ flux boundary condition. The number of particles per volume in inlet is computed via Eq3 3 imposing $D=D_{i n}$ and $\rho_{p}=k_{i n j} \rho_{\text {prop }}$, with $\rho_{\text {prop }}$ defined as the mean propellant density.

\section{Numerical Simulations}

In order to validate the aforementioned model, numerical simulations have been carried out. The presented computations have been provided by DIMA in-house code based on a finite volume technique and multi-blocks structured meshing. Conservative variables are calculated at the center of each computational cell whereas convective fluxes are computed at cell edges using an approximate Riemann solver (Roe scheme) for the gas phase and a donor-cell-type scheme for the particle phase. Space discretization is performed thanks to a second order TVD scheme, while second order accuracy in time is recovered using an explicit two-step Runge-Kutta time stepping.

The same SRM geometry presented in Gallier et al. [19] and Genot et al. [22] has been employed as test case since its tendency to be prone to thermoacoustic instability. The motor has a simple cylinder grain shape and it is short enough to prevent PVS [41]. In such a way no other form of instability would be able to develop but the thermoacoustic one. Concerning mesh properties, around 45, 000 cells are employed divided in two blocks with a strong refinement close to the upper edge where aluminum burning takes place.

\section{A. Numerical Results}

Both single-phase and multiphase simulations have been performed on the same geometry and with alike initial and boundary conditions. Flowfields are initialized using a nozzle seal in the throat whose rupture provides a transient wave system generation. Once steady-state condition is reached, a head-end velocity disturbance is enforced for a limited time range in order to trigger the possible onset of pressure fluctuations.

As preliminary results, simulations without particles have been carried out to assess the dynamic behaviour of the motor which, actually, does not show up any sort of instabilities and pressure oscillations after head-end forcing shutting-off.

Multiphase computation have been consequently run considering Duterque work [42] for condensed phase inlet: $k_{i n j}$ is assumed to be $0.06 \%$, whereas injection and residual particles diameters are respectively equal to $120 \mu m$ and $60 \mu \mathrm{m}$. Mean flowfields regarding pressure and combustion rate of condensed phase are reported in Fig 2 The head-end 
pressure value agrees with the one evaluated by the single-phase simulation whereas, as it was expected, the combustion zone of aluminum occurs in a very thin region close to the propellant grain surface since the high burning rate and the slow particles velocity involved. Temperature has continuously been checked to ensure that after aluminum particles combustion, gas temperature is equal to the single-phase solution value.

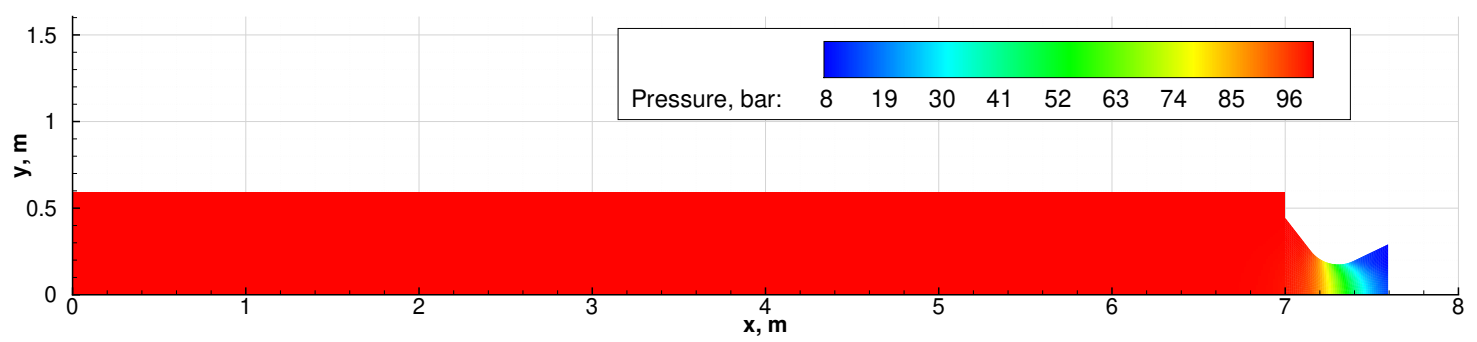

(a) Pressure flowfield.

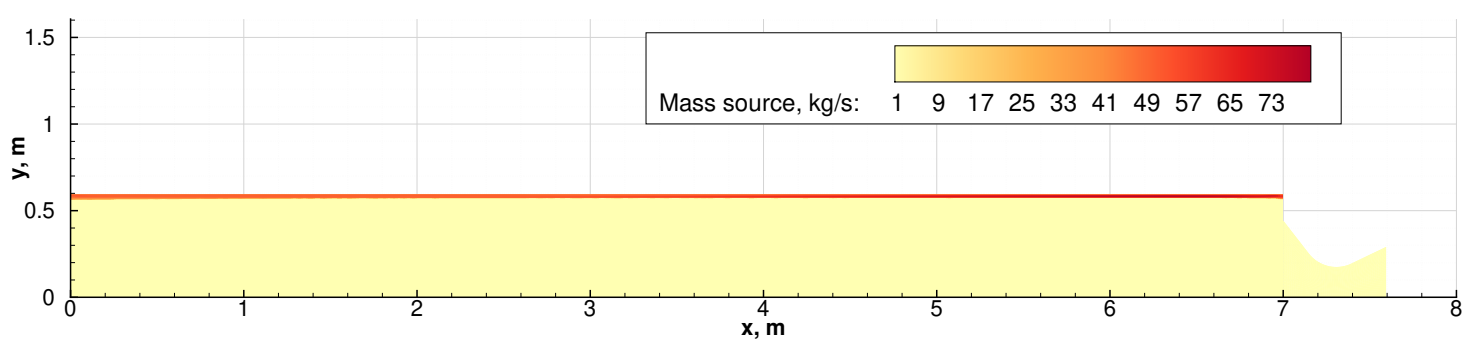

(b) Aluminum particles combustion rate flowfield.

Fig. 2 SRM mean flowfields.
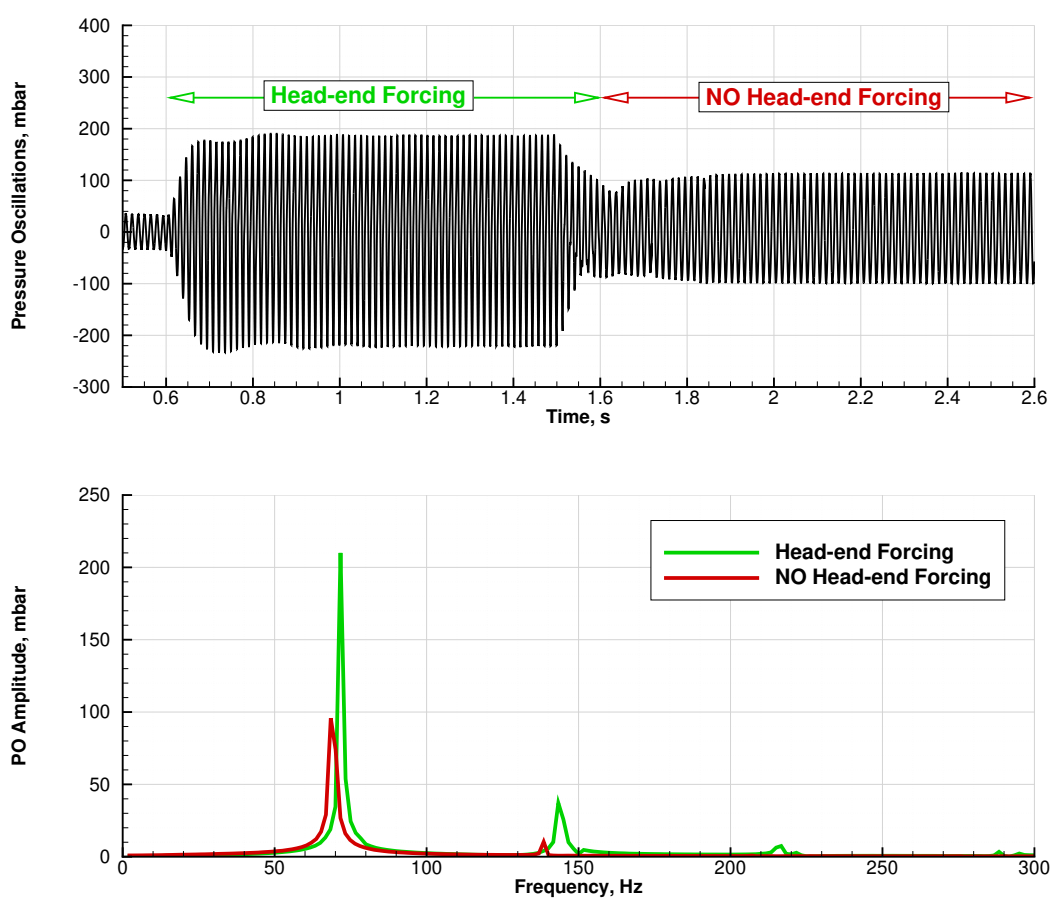

Fig. 3 Pressure oscillations and frequency content evaluated for multiphase simulation. 
On the contrary of the single-phase simulation, the two-phase reagent one shows up the occurrence of steady pressure fluctuations, as visible in Fig 3 also after switching off head-end forcing, with a frequency very close to the first acoustic longitudinal one, in plain accordance with references [19]. It is worth notice that such result is achieved thanks to a very simple physical multiphase modeling (just one class of particles injected and tracked) and the employment of Euler equations instead of Navier-Stokes one.

The thermoacoustics nature of such instability is confirmed analyzing the unsteady behaviour of the motor during the simulation last phase. In Fig 4 it is represented the unsteady vorticity distribution in the aft region. No vortices may be spotted, excluding the possibility of PVS or any other form of hydrodynamic instability. Indeed vorticity pattern suggests the presence of an acoustic boundary layer: the region close to the propellant wall characterized by the layered structure of the vorticity pattern [41, 43,-45]. Oscillatory vorticity (also known as shear wave) arises from the injection surface because of the viscoacoustic interaction in the unsteady flowfield. A slip flow associated with the irrotational acoustic motion is not allowed at the surface; the flow must enter the chamber in the radially inward direction with no axial component. This process inevitably produces vorticity, which is then convected downstream and dissipated by viscous effects. A consequence of such behaviour is represented by the intensification of velocity oscillations standing in the acoustic boundary layer with regard to the ones in regions far from the surface [19, 22, 45]. This last feature is also well recovered by the CFD solution hitherto presented as clearly visible in Fig 5

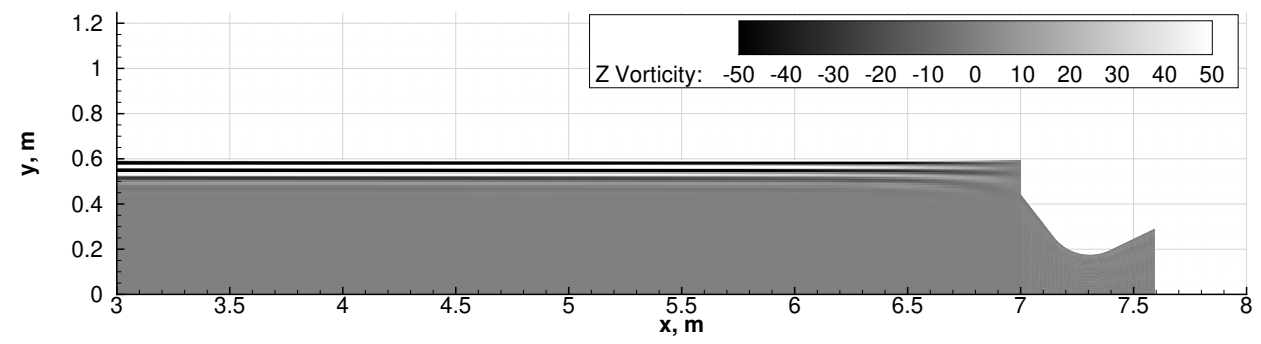

Fig. 4 Unsteady vorticity pattern in the motor rear part.

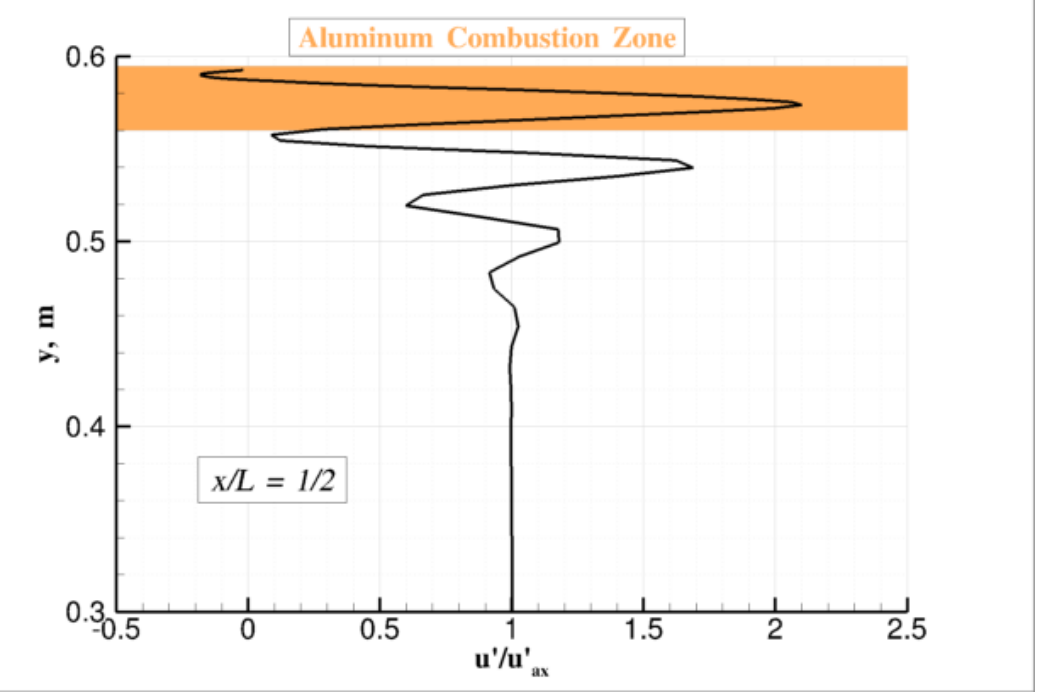

Fig. 5 Radial profile of axial acoustic velocity fluctuation.

The actual presence of the acoustic boundary layer, in the same region where combustion takes place, is crucial in order to establish a self sustained coupling between chamber acoustics and combustion. This last is actually controlled by velocity difference between gaseous and condensed phases thanks to convective effects assured by the Sherwood number of Eq6 Since particles inertia, in the acoustic boundary layer aluminum reactions may rely on a more powerful 
convective enhancement, burning more strongly each time the velocity fluctuation reaches a new peak. The first physical dissertation of such phenomenon was proposed by Raun and Beckstead [18] who assumed that significant coupling is likely to occur if oscillating flow causes an asymmetric disturbance in the flame, i.e., sweeping the flame envelope from side to side: acoustic driving would be enhanced by the large variation in flame area and hence, in heat release rate (Fig6).

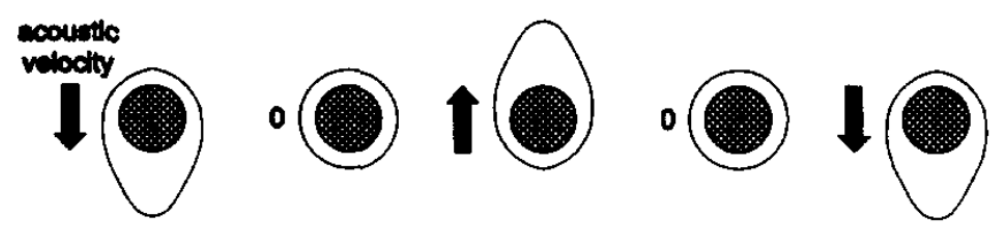

Fig. 6 Sketch of assumed physical mechanism behind thermnoacoustics instability in aluminum combustion [18].

It is worth to note that an acoustic boundary layer and consequently strong velocity fluctuations, may fully develop only where vortical structures, thus parietal vortex shedding, are not present [45]. However the presence of such feature does not suffice for thermoacoustics driven PO onset. In fact another remarkable point is related to aluminum combustion zone thickness and thus reaction time: as shown in Ref.[19], if the combustion is too slow, it will occur in a field fewer affected by strong velocity fluctuations, with the consequence of a possible lack of thermoacoustic feedback loop.

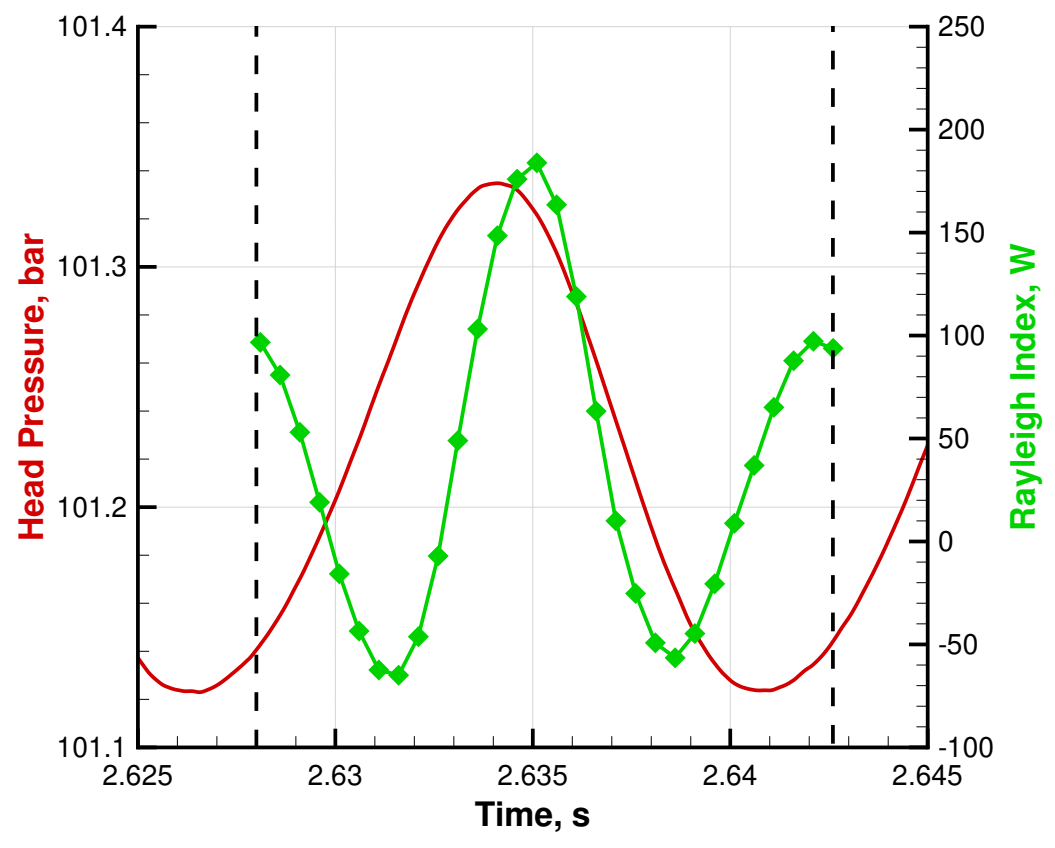

Fig. 7 Rayleigh index and head pressure as function of time.

In order to fully and definitely ensure the nature of the observed instability, an analysis on the Rayleigh criterion is performed, which determines the conditions for driving or suppressing flow oscillations when thermal energy is added periodically to the acoustic field [46]. It can be estimated by calculating the Rayleigh parameter, which is the time-averaged product between pressure and heat release fluctuations over one period. The global system is liable to be 
unstable if the following inequality is fulfilled:

$$
\int_{T} \int_{V} p^{\prime} q^{\prime} d V d t>0
$$

In Fig 7 it is reported the Rayleigh index integrated over the whole volume as function of time for one oscillation period. Notwithstanding the fluctuating behaviour it is easily proved that the time integral leads to an overall positive value: this definitely proves that the motor analyzed is prone to experience an instability via thermoacoustic coupling, matching the results presented in the reference works [19, 22].

\section{Conclusions}

This work aims to develop, introduce and validate a multiphase model focused on thermoacoustic instability in a in-house code currently used at DIMA. Such task is hopefully fulfilled trying to reduce the physical modeling tools necessary to address PO instability.

An Eulerian-Eulerian multiphase approach has been adopted together with an inviscid Euler description for the gaseous phase. A very simple combustion model for aluminum droplets has been exploited, carefully taking into account the convective effects on combustion rate.

The aforementioned model has been used for carrying out numerical simulations on simple cylinder grain SRM whose behaviour is referred to be instable in literature. Single-phase simulations have shown the motor to not be prone to any pressure oscillations. An opposite scenario occurs when multiphase modeling is set on, accordingly with literature data. The thermoacoustic nature of pressure fluctuations have been established thanks to Rayleigh criterion and flowfield analysis. Flow features, considered necessary to thermoacoustic instability to develop, are well recovered: the combustion region lies where the acoustic boundary layer actually is, enhancing pressure fluctuations due to the coupling between combustion rate and velocity oscillations.

These results represent an important starting point for future development of multiphase and thermoacoustics modeling and application at DIMA, enabling the possibility to reach a better and deeper understanding of pressure oscillations in SRMs. Indeed in the actual state-of-the-art, interaction between different forms of motor instability is not yet understood, a topic considered of primary importance since the massive employ of solid propulsion in the current and future scenario of European space transportation system.

\section{References}

[1] Culick, F., "Unsteady Motions in Combustion Chambers for Propulsion Systems," , 2006. RTO AGARD RTO-AG-AVT-039.

[2] Blomshield, F., Crump, J., Mathes, H., Stalnaker, R. A., and Beckstead, M., "Stability Testing of Full-Scale Tactical Motors," Journal of Propulsion and Power, Vol. 13, 2007, pp. 349-355. https://doi.org/10.2514/2.5191

[3] Ballereau, S., Godfroy, F., Guery, J. F., and Ribereau, D., "Assessment on Analysis and Prediction Method Applied on Thrust Oscillations of Ariane 5 Solid Rocket Motor," $39^{\text {th }}$ AIAA Joint Propulsion Conference, Huntsville, AL, 2003. AIAA Paper 2003-4675.

[4] Fabignon, Y., Dupays, J., Avalon, G., Vuillot, F., Lupoglazoff, N., Casalis, G., and Prévost, M., "Instabilities and pressure oscillations in solid rocket motors," Aerospace science and technology, Vol. 7, No. 3, 2003, pp. 191-200.

[5] Nesman, T., "RSRM Chamber Pressure Oscillations: Full Scale Ground and Flight Test Summary and Air Flow Test Results," AIAA Joint Propulsion Conference, 1995.

[6] Brown, R. S., Dunlap, R., Young, S. W., and Waugh, R. C., "Vortex Shedding as a Source of Acoustic Energy in Segmented Solid Rockets," Journal of Spacecrafts and Rockets, Vol. 18, No. 4, 1981, pp. 312-319.

[7] Dotson, K., Koshigoe, S., and Pace, K., "Vortex Shedding in a Large Solid Rocket Motor Without Inhibitors at the Segment Interfaces," Journal of Propulsion and Power, Vol. 13, No. 2, 1997, pp. 197-206.

[8] Blomshield, F., "Historical Perspective Of Combustion Instability In Motors-Case Studies,” 2001. https://doi.org/10.2514/6.20013875 AIAA Paper 2001-3875.

[9] Laureti, M., Favini, B., and Rossi, G., "P80 SRM Pressure Oscillations Reconstruction," AIAA Joint Propulsion Conference, Cincinnati, Ohio, 2018. 
[10] Larrieu, S., Godfroy, F., Orlandi, O., Robert, E., Ciucci, A., and Cesco, N., "Demonstration of Pressure Oscillation Reduction in the Frame of New Launchers Development," $6^{\text {th }}$ European Conference for AeroSpace Sciences, Krakov, Poland, 2015.

[11] Larrieu, S., "Demonstration of Pressure Oscillation Reduction For a New Large Monolithic Motor," Space Propulsion, Rome, Italy, 2016. Paper n. 3125096.

[12] French, A., Panelli, M., Di Lorenzo, G., Schettino, A., and Paglia, F., "Combustion Instability and Pressure Oscillation Numerical Simulation in a Solid Rocket Motor,” AIAA Joint Propulsion Conference, Atlanta, Georgia, 2017.

[13] Larrieu, S., Orlandi, O., Godfroy, F., and Di Trapani, C., “Two minutes inside P120C SRM,” Space Propulsion 2018, Seville, Spain, 2018.

[14] Price, E. W., and Sigman, R. K., "Combustion of Aluminized Solid Propellants," Solid Propellant Chemistry, Combustion, and Motor Interior Ballistics, Vol. 185, 2000, pp. 663-688.

[15] Beckstead, M. W., "A Summary of Aluminum Combustion,” VKI Special Course on Internal Aerodynamics in Solid Rocket Propulsion,, von Kármán Inst. Rept. RTO-EN-023, Rhode-Saint-Genèse, Belgium, 2003.

[16] King, M. K., "Prediction of Metal Combustion Efficiency in Low Port-to-Throat Area Ratio and Nozzleless Solid Rocket Motors," AIAA/SAE/ASME Joint Propulsion Conference, Cleveland, Ohio, USA, 1982.

[17] Kuo, K. K., Principles of Combustion, John Wiley \& Sons, New York, 1986.

[18] Raun, R. L., and Beckstead, M. W., "A numerical model for temperature gradient and particle effects on Rijke burner oscillations," Combustion and flame, Vol. 94, No. 1, 1993, pp. 1-24.

[19] Gallier, S., and Godfroy, F., "Aluminum combustion driven instabilities in solid rocket motors," Journal of Propulsion and Power, Vol. 25, No. 2, 2009, pp. 509-521.

[20] Gallier, S., Sibe, F., and Orlandi, O., "Combustion Response of an Aluminum Droplet Burning in Air," Proceedings of the Combustion Institute, Vol. 33, No. 2, 2011. https://doi.org/10.1016/j.proci.2010.05.046

[21] Gallier, S., Briquet, B., and Yiao, M., "Aluminum Combustion Can Drive Instabilities in Solid Rocket Motors: T-Burner Study," Journal of ropulsion and Power, Vol. 35, No. 1, 2019. https://doi.org/10.2514/1.B37034.

[22] Genot, A., Gallier, S., and Schuller, T., "Model for Acoustic Induced Aluminum Combustion Fluctuations in Solid Rocket Motors," Journal of ropulsion and Power, Vol. 35, No. 4, 2019. https://doi.org/10.2514/1.B37437

[23] Orlandi, O., Plaud, M., Godfroy, F., Larrieu, S., and Cesco, N., "Aluminium droplets combustion and SRM instabilities," Acta Astronautica, Vol. 158, 2019. https://doi.org/10.1016/j.actaastro.2019.03.036

[24] Laureti, M., and Favini, B., "Pressure Oscillations in aft-finocys SRMs," AIAA SciTech Forum, San Diego, California, 2019.

[25] Grossi, M., Laureti, M., and Favini, B., "Quasi-one Dimensional Model of Pressure Oscillations in Aft-Finocyl Solid Rocket Motors: a Critical Evaluation of Alternative Closure Sub-Models and Calibrations," AIAA Propulsion and Energy Forum, Indianapolis, IN, USA, 2019.

[26] Laureti, M., Favini, B., and Rossi, G., “Aeroacoustics of Aft-Finocyl Solid Rocket Motors,” AIAA Joint Propulsion Conference, Cincinnati, Ohio, 2018.

[27] Laureti, M., Rossi, G., Favini, B., Cavallini, E., and Neri, A., "CFD Study of Vorticity Patterns and Sound Generation in Simplified Aft-Finocyl Geometries,", EUCASS, $7^{\text {th }}$ European Conference for AeroSpace Sciences, 2017.

[28] Di Mascio, A., Cavallini, E., Favini, B., and Neri, A., "Vortex-Sound Generation and Thrust Unsteadiness in Aft-Finocyl Solid Rocket Motor," AIAA Joint Propulsion Conference, Cleveland, Florida, 2017.

[29] Attili, A., Favini, B., Di Giacinto, M., and Serraglia, F., "Numerical Simulation of Multiphase Flows in Solid Rocket Motors," AIAA/ASME/SAE/ASEE Joint Propulsion Conference and Exhibit, 2009.

[30] Marble, F. E., "Dynamics of a Gas Containing Small Solid Particles,” 5th AGARD Colloquium, 1963.

[31] Daniel, E., "Eulerian Approach for Unsteady Two-Phase Solid Rocket Flows with Aluminum Particles," Journal of Propulsion and Power, Vol. 16, 2000, pp. 309-317.

[32] Glassman, I., "Metal Combustion Processes," , 1959. American Rocket Society Preprint 938-59, New York. 
[33] Brzustowski, T. A., and Glassman, I., "Spectroscopic Investigation of Metal Combustion,” Heterogeneous Combustion, 1964, pp. 41-74.

[34] Law, C. K., "A Simplified Theoretical Model for the Vapor-Phase Combustion of Metal Particles," Combustion Science and Technology, Vol. 7, 1973, pp. 197-212.

[35] Hermsen, R. W., “Aluminum Combustion Efficiency in Solid Rocket Motors,” AIAA 19th Aerospace Sciences Meeting, St. Loius, MO, USA, 1981.

[36] Spalding, D. B., “Combustion of Fuel Particles,” Fuel, Vol. 30, No. 1, 1951, pp. 121-130.

[37] Liaw, P., and Chen, Y., "Advanced Multi-Phase Flow CFD Model Development for Solid Rocket Motor Flowfield Analysis,” , 1995. Nasa Final Report, Contract No. NAS8-39398.

[38] Crowe, C. T., Schwarzkopf, J. D., Sommerfeld, M., and Tsuji, Y., Multiphase Flows with Droplets and Particles, CRC Press, Taylor \& Francis Group, 6000 Broken Sound Parkway NW, Suite 300, 2012.

[39] Ranz, W. E., and Marshall, W. R., "Evaporation from drops," Chemical Engineering Progress, Vol. 48, No. 3, 1952, pp. 141-146.

[40] Clift, R., Grace, J. R., and Weber, M. E., Bubbles, Drops, and Particles, Academic Press, New York, 1978.

[41] Apte, S., and Yang, V., "Unsteady Flow Evolution in Porous Chamber with Surface Mass Injection, Part 1: Free Oscillation," AIAA Journal, Vol. 39, No. 8, 2001, pp. 1577-1586.

[42] Duterque, J., "Experimental Studies of Aluminum Agglomeration in Solid Rocket Motors," $4^{\text {th }}$ International Symposium on Special Topics in Chemical Propulsion, Begell House, Inc., 1996.

[43] Shaeffer, C. W., and Brown, R. S., "Oscillatory Internal Flow Fields Studies," , 1990. United Technologies, AFOSR Contract Report F04620-90-C-0032, San Jose, CA.

[44] Flandro, G. A., "Effects of Vorticity on Rocket Combustion Stability," Journal of Propulsion and Power, Vol. 11, No. 4, 1995.

[45] Apte, S., and Yang, V., "Unsteady Flow Evolution in Porous Chamber with Surface Mass Injection, Part 2: Acoustic Excitation," AIAA Journal, Vol. 40, No. 2, 2002, pp. 244-253.

[46] Rayleigh, J. W. S., “The Explanation of Certain Acoustical Phenomena,” Nature (London), Vol. 18, No. 455, 1878 , pp. 319-321. 